\title{
Adaptive Output Feedback Control Using Fault Compensation and Fault Estimation for Linear System with Actuator Failure
}

\author{
Jun Wang ${ }^{1} \quad$ Hai-Long Pei ${ }^{1,2} \quad$ Nai-Zhou Wang ${ }^{1}$ \\ ${ }^{1}$ Key Laboratory of Autonomous Systems and Networked Control, Ministry of Education, \\ South China University of Technology, Guangzhou 510640, China \\ ${ }^{2}$ College of Automation Science and Engineering, South China University of Technology, Guangzhou 510640, China
}

\begin{abstract}
The problem of linear systems subject to actuator faults (outage, loss of effectiveness and stuck), parameter uncertainties and external disturbances is considered. An active fault compensation control law is designed which utilizes compensation in such a way that uncertainties, disturbances and the occurrence of actuator faults are account for. The main idea is designing a robust adaptive output feedback controller by automatically compensating the fault dynamics to render the close-loop stability. According to the information from the adaptive mechanism, the updating control law is derived such that all the parameters of the unknown input signal are bounded. Furthermore, a disturbance decoupled fault reconstruction scheme is presented to evaluate the severity of the fault and to indicate how fault accommodation should be implemented. The advantage of fault compensation is that the dynamics caused by faults can be accommodated online. The proposed design method is illustrated on a rocket fairing structural-acoustic model.
\end{abstract}

Keywords: Fault compensation, fault-tolerant control, robust adaptive control, fault reconstruction, actuator failure.

\section{Introduction}

Fault-tolerant control (FTC) has the ability of keeping the system reliability with performance degradation, which can be accepted when it is affected by a fault. However, if an actuator is suddenly stuck and can no longer deflect a certain control surface in an aircraft, or the system performance is not accepted by the happened faults and/or unknown external disturbance, these faults will lead to catastrophic events. So the research on accommodating actuator failures and maintaining acceptable system performance has received considerable attention from control engineers. Survey papers such as [1] highlighted recent work in the area of FTC and discussed the various application areas.

In general, FTC can be classified into two different categories: Passive control and active control. The passive control uses a fixed controller to deal with any faultfree and faulty cases ${ }^{[2-5]}$. Therefore, this kind of control cannot guarantee the system's stability when a fault exceeds the predefined faulty set. Unlike the passive control, the active control can compensate for faults by predefining control law or by detecting fault information, location and size for control allocation (CA) strategy online, so that stability and some required performance of the system are achieved in the presence of a fault. In the literature, there have been previous works about successfully implemented active control, including fault detection filterbased approach ${ }^{[6-9]}$, control allocation algorithm ${ }^{[10-12]}$, and observer-based approach ${ }^{[13-15]}$. For the fault type of loss of actuator, adaptive fault-tolerant control design

Manuscript received October 16, 2012; revised March 1, 2013

This work was supported by National Natural Science Foundation of China (No. 61174053) and the Specialized Research Fund for the Doctoral Program of Higher Education of China (No. 20100172110023). approaches were presented by estimating the actuator efficiency factor online ${ }^{[16-18]}$. Whereas some direct adaptive fault-tolerant control methods were applied to the actuator stuck faults ${ }^{[19,20]}$. Moreover, more general fault models including outage, loss of effectiveness and stuck we studied ${ }^{[21-23]}$, but no disturbance and/or parameter uncertainty have been considered. Recently, based on optimal technology, the self-restore method was used in fault tolerant control. A fault-tolerant control law for systems with measurement delays were developed ${ }^{[24]}$ by observer-based reduced-order fault diagnosis and dynamic self-restore technology, while by solving the Riccati equation and Sylvester equation, an optimal fault-tolerant control law was designed ${ }^{[25]}$.

When online faults can be estimated and decoupled with disturbances, the waveform of unknown input signal can be used to evaluate the severity of the fault and external disturbance, and indicate how fault accommodation should be implemented. Accordingly, the fault estimation techniques and disturbance decoupling techniques are applied to reconstructing the faults and disturbances. For some important parameters resulting in the faults, such as loss of actuator effectiveness, the adaptive observer can be used to estimate the unknown parameters ${ }^{[26-28]}$. Furthermore, the faults are regarded as additive unknown input, and an observer-based method with augmented vector, including the states and unknown faults, can be used to estimate the faults ${ }^{[29-31]}$.

The main contribution of this paper is the application of robust adaptive fault tolerant compensation control for linear systems subject to time-varying parameter uncertainties, unknown external disturbances and actuator faults. A new scheme, which is different from the sliding mode ob- 
server (SMO) based approach, is proposed to reconstruct the faults and disturbances by using Lyapunov redesign technique when the Hautus condition is not satisfied. By assuming some unknown variables, such as lower and upper bounds of fault factor, the upper bounds of disturbance and parameter uncertainty, the designed adaptive laws for system response errors and observer errors can adaptively adjust the controller's parameters to estimate these unknown lower or upper bounds and decouple the faults. The resulting controller can guarantee the closed-loop system to be uniformly bounded and asymptotically stable. Based on the adaptive output feedback controller and disturbance decoupled fault, the reconstruction scheme can be isolated from the faults and disturbances, and then the output feedback control is more advantageous to realize in practical projects, which is the biggest difference to [22].

The outline of this paper is as follows. The control objectives of this work and preliminary results are presented in Section 2. Section 3 provides a detailed description of the adaptive fault-tolerant controller design approach. In Section 4, the disturbance decoupled fault reconstruction scheme is presented. Numerical examples and simulation results are given in Section 5. And finally, we draw some concluding remarks in Section 6 .

Notation. For a real square matrix $A, \lambda_{\min }(A)$ and $\lambda_{\max }(A)$ denote the minimal and maximal eigenvalues of matrix $A, A>0(A<0)$ represents a symmetric positive (negative) definite matrix, and $A^{\mathrm{T}}$ denotes its transpose. Symbol $I_{\mathrm{n}}$ represents a $n$-th order identity matrix. $\mathbf{R}$ denotes the set of real numbers. A block diagonal matrix with matrices $X_{1}, X_{2}, \cdots, X_{n}$ on its main diagonal is denoted as $\operatorname{diag}\left\{X_{1}, X_{2}, \cdots, X_{n}\right\} \cdot\|\cdot\|$ represents the Euclidean norm of vectors or matrices.

\section{Problem formulation}

In this paper, we consider a stabilizable plant where a fault is associated with the actuators and assume that parameter uncertainty and external disturbances exist in the system, which can be shown as

$$
\begin{aligned}
& \dot{x}(t)=(A+\Delta A(t)) x(t)+B u_{f}(t)+B_{d} d(t) \\
& y(t)=C x(t)
\end{aligned}
$$

where $x(t) \in \mathbf{R}^{n}$ is the state vector, $u_{f}(t) \in \mathbf{R}^{n_{u}}$ is the control input, $d(t) \in \mathbf{R}^{n_{d}}$ models a bounded external disturbance, $A, B$ and $B_{d}$ are known matrices of compatible dimensions, and $\Delta A(t)$ represents the time-varying parameter uncertainty and is a diagonal matrix. $B_{d}$ and $\Delta A(t)$ are assumed to satisfy the following matching condition:

$$
\begin{aligned}
& B_{d}=B F_{d} \\
& \Delta A(t)=B S(t)
\end{aligned}
$$

where $F_{d}$ is a known matrix, and $S(t)$ denotes an unknown matrix that is bounded in the Euclidean norm $\|S(t)\| \leqslant l^{*}$ with $l^{*}$ being an unknown positive scalar.

Motivated by the need to devise schemes with guaranteed fault tolerance properties, the actuator fault model is considered including outage, loss of effectiveness, stuck con- dition or combination of all. Here, $u_{f}(t)$, which represents the output signal, contains all kinds of possible actuator faults and acts on the system directly, $u(t)$ present the actual input signal of the actuator. Then, a general actuator fault model is given as

$$
u_{f}(t)=\rho(t) u(t)+\Theta(t)
$$

where $\rho(t)=\operatorname{diag}\left\{\rho_{i}^{j}(t)\right\}$ is a diagonal matrix with $\rho_{i}^{j}(t) \in$ $[0,1](i=1,2, \cdots, m, j=1,2, \cdots, N)$ being the unknown time-varying scalar function called actuator efficiency factor, index $j$ denotes the $j$-th faulty mode, $N$ is the number of total faulty modes, and $\Theta(t)$ is the bounded timevarying function reflecting the actuator fault that is completely stuck or partially out of control. In order to explain the possible combination of $\rho$ and $\Theta$, we consider the following four cases:

1) $\rho(t)=1$ and $\Theta(t)=0$ : In this case, $u_{f}(t)=u(t)$. This indicates a healthy actuator.

2) $\rho(t) \neq 0$ and $\Theta(t) \neq 0: \rho(t)<1$ indicates that $u_{f}(t)$ can be partially influenced by the control input $u(t)$, which is partly effective to the actuators at unparameterisable bounded time-varying function $\Theta(t)^{[20]}$.

3) $\rho(t) \neq 0$ and $\Theta(t) \geqslant 0$ : When $\Theta(t)=0$, this case corresponds to the actuator's partial loss of effectiveness. When $\Theta(t)>0$ and is a constant, this case indicates partially stuck and adjustable for actuator failures.

4) $\rho(t)=0$ and $\Theta(t) \geqslant 0$ : When $\Theta(t)=0$, this case corresponds to outage ${ }^{[32]}$. When $\Theta(t)>0$, this case, which is uncontrollable, corresponds to completely stuck or out of control.

Remark 1. The second case implies that the parts of actuators are out of control, but the suck fault and loss of effectiveness may act on some actuators at the same time. The third case gives a special condition for the actuator fault. If $\Theta(t)$ is a constant control input, it can be considered as external disturbance. Once the fourth case happens, this system will become uncontrollable, any controller is meaningless.

The dynamics of system (1) with actuator fault modeled by (3) can then be written as

$$
\begin{aligned}
& \dot{x}(t)=(A+\Delta A(t)) x(t)+B \rho(t) u(t)+B \Theta(t)+B_{d} d(t) \\
& y(t)=C x(t) .
\end{aligned}
$$

Remark 2. For system (4), if $\Delta A(t)=0$, the fault tolerant state feedback control designed in [22] can ensure the system states to asymptotically converge to zero under the assumption that the lower and upper bounds of $\rho$ are known for each fault model. The constraint value for $\rho$ is too conservative. In this paper, the designed adaptive fault-tolerant control can guarantee that the states asymptotically converge to zero, despite the unknown bounds of $\rho$ and the existence of parameter uncertainty.

In order to illustrate a feasible fault-tolerant control (provided that $\rho(t) \neq 0$ ), we introduce the following assumptions.

Assumption 1. $\{A, B \rho\}$ is completely controllable and $\{C, A\}$ is completely observable for any actuator failure model $\rho \in\left\{\rho^{1}, \rho^{2}, \cdots, \rho^{N}\right\}$ under consideration. 
Assumption 2. The uncertainty actuator fault and unknown external disturbances are piecewise continuous bounded functions, i.e., there exist positive upper bounds $\bar{\kappa}_{\Theta}$ and $\bar{\kappa}_{d}$ such that

$$
\|\Theta(t)\| \leqslant \bar{\kappa}_{\Theta}<\infty, \quad\|d(t)\| \leqslant \bar{\kappa}_{d}<\infty .
$$

Assumption 3. $\operatorname{rank}[B \rho]=\operatorname{rank}[B]$ ( $\rho$ is not equal to zero) holds for any $\rho \in\left\{\rho^{1}, \rho^{2}, \cdots, \rho^{N}\right\}$.

Assumption 4. For any stuck or outage fault, if the remaining actuators can still achieve a designed control objective, then there exists a positive constant $\tau$ such that

$$
\left\|B^{\mathrm{T}} P x\right\|^{2} \leqslant \tau\left\|B^{\mathrm{T}} P x \sqrt{\rho}\right\|^{2} .
$$

Remark 3. It is well known that Assumption 1 stands for output-feedback design and denotes the internal stabilizability of any normal or fault system. Assumption 2 is common in the robust fault-tolerant control literature ${ }^{[22]}$. As discussed in [19], Assumption 3 introduces a necessary condition for completely compensating the stuck-actuator faults and disturbances. From [19,21-23], Assumption 4 proposes a constraint set on the actuation fault that can ensure the controllability of the plant and obtain a feasible solution for the actuator failure compensation problem.

Consider system (1), based on Assumption 1, $\{A, B\}$ is stabilizable and $\{C, A\}$ is detectable. Thus, there exists a $n_{u} \times m$ constant matrix $K$ such that matrix $A-B K C$ is Hurwitz. Then, for any $n$-th order positive definite symmetric matrix $Q$, the Lyapunov equation

$$
(A+B K C)^{\mathrm{T}} P+P(A+B K C)=-Q
$$

has a unique positive definite symmetric solution $P \in$ $\mathbf{R}^{n \times n}$. Furthermore, Assumption 3 guarantees that the linear combinations of the columns in $B$ can be reconstructed by $B \rho$ for any fault, i.e., there exists a $K_{1}$ such that

$$
\left(A+B \rho K_{1} C\right)^{\mathrm{T}} P+P\left(A+B \rho K_{1} C\right)=-Q
$$

where $K_{1}$ is the estimation of the feedback gain $K$. Then, according to Assumptions 1-4, the main purpose of this paper is to construct a robust adaptive compensation controller to guarantee the closed-loop stability in the presence of faults.

\section{Robust adaptive fault-tolerant con- troller design}

To achieve the desired control objective given in Section 2 , the adaptive controller is designed in the form of

$$
u(t)=\hat{K}_{1} y(t)+u_{a}(t)+u_{b}(t)
$$

where $\hat{K}_{1}(t)=\left[\hat{K}_{11}(t), \hat{K}_{12}(t), \cdots, \hat{K}_{1 n_{u}}(t)\right]^{\mathrm{T}} \in \mathbf{R}^{n_{u} \times m}$ is the estimation of unknown matrix $K_{1}$ in (6) and is updated by the adaptive law as

$$
\frac{\mathrm{d} \hat{K}_{1 i}(t)}{\mathrm{d} t}=-\Pi_{i} x x^{\mathrm{T}} P b_{i}, i=1,2, \cdots, n_{u}
$$

where $\Pi_{i}$ is any positive constant, $b_{i}$ is the $i$-th column of $B, P$ is defined as in (5) and $(6)$, and $\hat{K}_{1 i}(t)$ is finite. $u_{a}(t)$ and $u_{b}(t)$ are two auxiliary compensation control functions which will be given later.

With the proposed control (4), one has

$$
\begin{aligned}
\dot{x}(t)= & (A+\Delta A(t)) x(t)+B \rho\left(\hat{K}_{1} y(t)+u_{a}(t)+\right. \\
& \left.u_{b}(t)\right)+B \Theta(t)+B_{d} d(t) .
\end{aligned}
$$

By Assumption 2, there also exists a positive constant $k_{1}$ satisfying

$$
\left\|\Theta(t)+F_{d} d(t)\right\| \leqslant \kappa_{\Theta}+\left\|F_{d}\right\| \kappa_{d} \leqslant \tau k_{1} .
$$

Here, it is worth pointing out that constants $\kappa_{\Theta}, \kappa_{d}, \tau$ and $k_{1}$ are all unknown.

Now, we give the auxiliary control functions $u_{a}(t)$ and $u_{b}(t)$ as

$$
\begin{gathered}
u_{a}(t)=\frac{-\hat{k}_{1} B^{\mathrm{T}} P x}{\left\|B^{\mathrm{T}} P x\right\|+\varepsilon \mathrm{e}^{-\sigma t}} \\
u_{b}(t)=-0.5 k_{2} B^{\mathrm{T}} P x
\end{gathered}
$$

where $\hat{k}_{1}$ is the estimation of $k_{1}, \varepsilon$ and $\sigma$ are positive constants, $k_{2}$, which will be given later, is a constant. We define that the estimation of $\hat{k}_{1}$ is updated by the adaptive law as

$$
\frac{\mathrm{d} \hat{k}_{1}}{\mathrm{~d} t}=2 \gamma\left\|B^{\mathrm{T}} P x\right\|
$$

where $\gamma$ is positive constant and $\hat{k}_{1}\left(t_{0}\right)$ is finite.

Denote $\tilde{K}_{1}(t)=\hat{K}_{1}(t)-K_{1}$ and $\tilde{k}_{1}(t)=\hat{k}_{1}(t)-k_{1}$. Due to the fact that $K$ and $k_{1}$ are unknown constants, we can write the error system as

$$
\frac{\mathrm{d} \tilde{K}_{1 i}(t)}{\mathrm{d} t}=-\Pi_{i} x x^{\mathrm{T}} P b_{i}, \frac{\mathrm{d} \tilde{k}_{1}}{\mathrm{~d} t}=2 \gamma\left\|B^{\mathrm{T}} P x\right\| .
$$

In the following, by $\left(x, \tilde{K}_{1}, \tilde{k}_{1}\right)$, we denote a solution of the closed-loop system and the error system. Then, we can obtain the following theorem.

Theorem 1. Considering the adaptive closed-loop system (9) and error system (14) under Assumptions 1-4, there exist solutions to the closed-loop system and error system, which are asymptotically stable by using designed adaptive fault tolerant control.

Proof. For the adaptive closed-loop system described by (13), we first define a Lyapunov function candidate as

$$
V=x^{\mathrm{T}} P x+\sum_{i=1}^{n_{u}} \rho_{i} \tilde{K}_{1 i}^{\mathrm{T}} \Pi_{i}^{-1} \tilde{K}_{i}+\frac{1}{2} \tau \gamma^{-1} \tilde{k}_{1}^{2} .
$$

Then, according to (14) and Assumption 4, the time deriva- 
tive of $V$ for $t>0$ is

$$
\begin{aligned}
\dot{V}= & 2 x^{\mathrm{T}} P\left(\left(A+\Delta A+B \rho \hat{K}_{1} C\right) x+B \rho\left(u_{a}+u_{b}\right)+\right. \\
& B\left(\Theta+F_{d} d\right)+2 \sum_{i=1}^{n_{u}} \rho_{i} \tilde{K}_{1 i}^{\mathrm{T}} \Pi_{i}^{-1} \dot{\tilde{K}}_{1 i}+\tau \gamma^{-1} \tilde{k}_{1} \dot{\tilde{k}}_{1} \leqslant \\
& x^{\mathrm{T}}\left(P\left(A+B \rho \hat{K}_{1} C\right)+\left(A+B \rho \hat{K}_{1} C\right)^{\mathrm{T}} P\right) x+ \\
& 2\left\|x^{\mathrm{T}} P B\right\|\|S(t)\|\|x\|-\frac{2 x^{\mathrm{T}} P B \rho B^{\mathrm{T}} P x \hat{k}_{1}}{\left\|x^{\mathrm{T}} P B\right\|+\varepsilon \mathrm{e}^{-\sigma t}-} \\
& k_{2} x^{\mathrm{T}} P B \rho B^{\mathrm{T}} P x+2\left\|x^{\mathrm{T}} P B\right\|\left(\kappa_{\Theta}+\|F\| \kappa_{d}\right)+ \\
& 2 \sum_{i=1}^{n_{u}} \rho_{i} \tilde{K}_{1 i}^{\mathrm{T}} \Pi_{i}^{-1} \dot{\tilde{K}}_{1 i}+\tau \gamma^{-1} \tilde{k}_{1} \dot{\tilde{k}}_{1} \leqslant \\
& x^{\mathrm{T}}\left(P\left(A+B \rho \hat{K}_{1} C\right)+\left(A+B \rho \hat{K}_{1} C\right)^{\mathrm{T}} P\right) x+ \\
& 2 l^{*}\left\|x^{\mathrm{T}} P B\right\|\|x\|-\frac{2 \tau\left\|x^{\mathrm{T}} P B\right\|^{2} \hat{k}_{1}}{\left\|x^{\mathrm{T}} P B\right\|+\varepsilon \mathrm{e}^{-\sigma t}-} \\
& k_{2} \tau\left\|x^{\mathrm{T}} P B\right\|^{2}+2\left\|x^{\mathrm{T}} P B\right\| \tau k_{1}+ \\
& 2 \sum_{i=1}^{n_{u}} \rho_{i} \tilde{K}_{1 i}^{\mathrm{T}} \Pi_{i}^{-1} \dot{\tilde{K}}_{1 i}+\tau \gamma^{-1} \tilde{k}_{1} \dot{\tilde{k}}_{1} \leqslant \\
& x^{\mathrm{T}}\left(P\left(A+B \rho \hat{K}_{1} C\right)+\left(A+B \rho \hat{K}_{1} C\right)^{\mathrm{T}} P\right) x+ \\
& \tau \frac{l^{* 2}}{\tau}\left\|x^{\mathrm{T}} P B\right\|^{2}+\|x\|^{2}-\frac{2 \tau\left\|x^{\mathrm{T}} P B\right\|^{2} \hat{k}_{1}}{\left\|x^{\mathrm{T}} P B\right\|+\varepsilon \mathrm{e}^{-\sigma t}-} \\
& k_{2} \tau\left\|x^{\mathrm{T}} P B\right\|^{2}+2\left\|x^{\mathrm{T}} P B\right\| \tau k_{1}+ \\
& 2 \sum_{i=1}^{n_{u}} \rho_{i} \tilde{K}_{1 i}^{\mathrm{T}} \Pi_{i}^{-1} \dot{\tilde{K}}_{1 i}+2 \tau \tilde{k}_{1}\left\|x^{\mathrm{T}} P B\right\| . \\
& (16) \\
&
\end{aligned}
$$

Let's use (6) and define $k_{2}=\frac{l^{* 2}}{\tau}$, and the above equation can be simplified as

$$
\begin{aligned}
\dot{V} \leqslant & x^{\mathrm{T}}(-Q+I) x+2 x^{\mathrm{T}} P B \rho \tilde{K} x+2 \sum_{i=1}^{n_{u}} \rho_{i} \tilde{K}_{i}^{\mathrm{T}} \Pi_{i}^{-1} \dot{\tilde{K}}_{i}+ \\
& \frac{2 \tau\left\|x^{\mathrm{T}} P B\right\| \hat{k}_{1} \varepsilon \mathrm{e}^{-\sigma t}}{\left\|x^{\mathrm{T}} P B\right\|+\varepsilon \mathrm{e}^{-\sigma t}}
\end{aligned}
$$

Define a new variable $\Gamma$ which is also a positive symmetric definite matrix and $\Gamma=Q-I$. Then, (5) can be revised to $(A+B K C)^{\mathrm{T}} P+P(A+B K C)=-\Gamma$. Considering the adaptive law given in (8), we have

$$
2 x^{\mathrm{T}} P B \rho \tilde{K} x+2 \sum_{i=1}^{n_{u}} \rho_{i} \tilde{K}_{i}^{\mathrm{T}} \Pi_{i}^{-1} \dot{\tilde{K}}_{i}=0 .
$$

Then, it follows that

$$
\dot{V} \leqslant-x^{\mathrm{T}} \Gamma x+\frac{2 \tau\left\|x^{\mathrm{T}} P B\right\| \hat{k}_{1} \varepsilon \mathrm{e}^{-\sigma t}}{\left\|x^{\mathrm{T}} P B\right\|+\varepsilon \mathrm{e}^{-\sigma t}}, \forall t \geqslant 0 .
$$

Since

$$
\frac{2 \tau\left\|x^{\mathrm{T}} P B\right\| \hat{k}_{1} \varepsilon \mathrm{e}^{-\sigma t}}{\left\|x^{\mathrm{T}} P B\right\|+\varepsilon \mathrm{e}^{-\sigma t}} \leqslant\left\{\begin{array}{l}
0, x(t) \rightarrow 0 \\
2 \tau \hat{k}_{1} \varepsilon \mathrm{e}^{-\sigma t}, x(t) \neq 0
\end{array}\right.
$$

$\dot{V}$ satisfies that

$$
\dot{V} \leqslant-x^{\mathrm{T}} \Gamma x+c \mathrm{e}^{-\sigma t} \leqslant-\lambda\|x\|^{2}+c \mathrm{e}^{-\sigma t}
$$

where $\lambda \triangleq\left\{\frac{\lambda_{\min }(\Gamma)}{\left(\lambda_{\max }(P)\right)}\right\}>0$. The $2 \tau \hat{k}_{1} \varepsilon$ can be replaced by a constant $c$. Further, $V(t)$ can be shown as

$$
\begin{gathered}
0 \leqslant V(t) \leqslant V\left(t_{0}\right)-\int_{t_{0}}^{t} \lambda\|x(\delta)\|^{2} \mathrm{~d} \delta+\int_{t_{0}}^{t} c \mathrm{e}^{-\sigma \delta} \mathrm{d} \delta \\
\forall t_{0} \geqslant 0
\end{gathered}
$$

For the second term of the right-hand side of (21), it can be easily verified that $\int_{t_{0}}^{t} c \mathrm{e}^{-\sigma \delta} \mathrm{d} \delta \rightarrow 0$, when $t \rightarrow \infty$.

For (21), we can get the following form

$$
\lim _{t \rightarrow \infty} \int_{t_{0}}^{t} \lambda\|x(\delta)\|^{2} \mathrm{~d} \delta \leqslant V\left(t_{0}\right) .
$$

Because $x(t)$ is uniformly bounded, which implies $x(t)$ is uniformly continuous, $\lambda\|x(\delta)\|^{2}$ is also uniformly continuous. Applying the Barbalat Lemma ${ }^{[33]}$ to (22) leads to the result

$$
\lim _{t \rightarrow \infty} \int_{t_{0}}^{t} \lambda\|x(\delta)\|^{2} \mathrm{~d} \delta=0
$$

which means the closed-loop system is asymptotically stable.

Remark 4. Robust FTC refers to the ability of the fault-tolerant control scheme to avoid invalid reconfiguration in the presence of modeling uncertainties and disturbances. It was reported that an output feedback composite controller was designed which was different from a state feedback controller ${ }^{[2]}$ and was simple to use in practice. However, it is undeniable that the selected $\Gamma$ was conservative and the results could not be influenced by it.

Remark 5. The auxiliary control function $u_{a}(t)$, described by (11), shows the actuator failure without the knowledge of the lower bound of actuator efficiency factor. And then the amended adaptive law $\varepsilon \mathrm{e}^{-\sigma t}$ is different from $\sigma(t)$ in [34], and it can also validly avoid the high gain problem, while the high gain problem arises from the lower bound of $\rho(t)$ is too small.

\section{Perturbation decoupled fault recon- figuration}

In Section 3, the combined effects of fault dynamics and disturbances were considered. Now we propose a reconfiguration approach for disturbance decoupled in the observer system. By using the Hautus condition ${ }^{[35]}$, if $\operatorname{rank}(C \bar{B})=q$ is satisfied, where $\bar{B}=\left[B, B_{d}\right]$, a disturbance observer can be structured to estimate the external signal. Hence, we can suppose that there exist matrices $P>0, Q>0$ and observer gain $L$, satisfying

$$
P(A-L C)+(A-L C)^{\mathrm{T}} P=-Q<0 .
$$

Then, we consider an output error equation with weighting matrix $W$ as

$$
e(t)=W(C x-C \hat{x})
$$

where $\hat{x}$ stands for the estimation of state $x$. The weighting matrix $W$ should have $q$ rows. We suppose that $P \bar{B}=$ $(W C)^{\mathrm{T}}$, where $P$ is the same as (24). Therefore, according to the Kalman-Yakubovich-Popov (KYP) lemma, the $\{A-$ 
$L C, \bar{B}, W C, 0\}$ is a minimal realization of $G(s)$ and $G(s)$ is strictly positive real $(\mathrm{SPR})^{[36]}$. The above condition is often adopted as an assumption in SMC-based fault estimation system $^{[13]}$. But, if $C \bar{B}$ does not have full column rank, then the observer and fault detection filter method cannot satisfy the disturbance decoupling requirement based on unknown input.

In Section 2, $u_{f}(t)=\rho u(t)+\Theta(t)$, here, we define a new variable $\zeta$ satisfying $\rho=1-\zeta$. Then, $u_{f}(t)=u(t)-u_{f}^{*}(t)$, where $u_{f}^{*}(t)=\zeta u(t)-\Theta(t)$. System equation (1) can be amended in the form of

$$
\begin{aligned}
& \dot{x}(t)=(A+\Delta A(t)) x(t)+B\left(u(t)-u_{f}^{*}(t)+F_{d} d(t)\right) \\
& y=C x(t) .
\end{aligned}
$$

And then, the observer is builded as

$$
\begin{aligned}
& \dot{\hat{x}}(t)=(A+\Delta \mathrm{A}) \hat{x}(t)+B\left(u(t)+u_{\mathrm{des}}(t)\right)+L(y(t)-\hat{y}(t)) \\
& \hat{y}=C \hat{x}(t) \\
& e(t)=W(y(t)-\hat{y}(t))
\end{aligned}
$$

where $u_{\text {des }}$ is designed as a continuous function of $e(t)$ in order to reconstruct $-u_{f}^{*}(t)+F_{d} d(t)$. To decouple the disturbance, matrices $W \in \mathbf{R}^{l \times m}$ and $L$ are required to satisfy the following assumptions.

Assumption 5. There exist matrices $W$ and $L$ so that $A-L C$ is Hurwitz. And $W C B_{d}=0, W C B$ has full column rank. The magnitude of $u_{f d}=-u_{f}^{*}(t)+F_{d} d(t)$ is bounded by a continuous known function $\alpha_{f d}$ as $\left\|\alpha_{f d}\right\| \leqslant \beta(t)$.

Assumption 6. For the designed $u_{\text {des }}$ and any finite $T>0$, the following equation is satisfied

$$
\lim _{t \rightarrow 0} \int_{t}^{t+T} \beta(\delta)-u_{\mathrm{des}}(\delta) \mathrm{d} \delta=0, \forall 0<T<+\infty .
$$

Theorem 2. Consider system (1) under Assumptions 5-6 and denote $\Lambda=\operatorname{diag}\left\{\lambda_{1}, \lambda_{2}, \cdots, \lambda_{l}\right\}$, where $\lambda_{i}$ is the eigenvalue of $A-L C$. The disturbance decoupled fault reconstruction system (27) using

$$
u_{\mathrm{des}}=\frac{\beta(t)^{2} H e(t)}{\beta(t)\|H e(t)\|+\varepsilon \mathrm{e}^{-\sigma t}}+\frac{k_{3}\left\|e(t)^{\mathrm{T}} P B\right\|^{2} H e(t)}{\|H e(t)\|^{2}+\varepsilon \mathrm{e}^{-\sigma t}}
$$

satisfies $e(t) \rightarrow 0$ as $t \rightarrow \infty$, where $H=(P W C B)^{\mathrm{T}}$ and $P$ is a positive definite matrix satisfying

$$
\Lambda^{\mathrm{T}} P+P \Lambda<0
$$

Proof. Denote $\xi=x(t)-\hat{x}(t)$ and $e(t)=W C \xi(t)$. According to Assumption 5, $e(t)$ satisfies the dynamics as

$$
\begin{aligned}
\dot{e}= & W C(A+\Delta A-L C) \xi+W C B\left(u_{f d}-u_{\mathrm{des}}\right)= \\
& (\Lambda+\Delta A) W C \xi+W C B\left(u_{f d}-u_{\mathrm{des}}\right)= \\
& (\Lambda+\Delta A) e+W C B\left(u_{f d}-u_{\mathrm{des}}\right) .
\end{aligned}
$$

We choose the Lyapunov function $V=e(t)^{\mathrm{T}} P e(t)$ for the above system. Thus,

$$
\begin{aligned}
\dot{V}= & 2 e(t)^{\mathrm{T}} P\left((\Lambda+\Delta A) e(t)+W C B\left(u_{f d}-u_{\text {des }}\right)\right)= \\
& -e(t)^{\mathrm{T}}\left(P \Lambda+\Lambda^{\mathrm{T}} P\right) e+2 l^{*}\left\|e(t)^{\mathrm{T}} P B\right\|\|e(t)\|+ \\
& 2 e(t)^{\mathrm{T}} P W C B\left(u_{f d}-u_{\text {des }}\right) \leqslant \\
& -e(t)^{\mathrm{T}}\left(P \Lambda+\Lambda^{\mathrm{T}} P\right) e(t)+\|e(t)\|^{2}+l^{* 2}\left\|e(t)^{\mathrm{T}} P B\right\|^{2}+ \\
& 2(H e(t))^{\mathrm{T}}\left(u_{f d}-u_{\text {des }}\right) \leqslant \\
& -e(t)^{\mathrm{T}}\left(P \Lambda+\Lambda^{\mathrm{T}} P\right) e(t)+l^{* 2}\left\|e(t)^{\mathrm{T}} P B\right\|^{2}+ \\
& 2 \beta(t)\|H e(t)\|-\frac{2 \beta(t)^{2}(H e(t))^{\mathrm{T}} H e(t)}{\beta(t)\|H e(t)\|+\varepsilon \mathrm{e}^{-\sigma t}-} \\
& \frac{k_{3}\left\|e(t)^{\mathrm{T}} P B\right\|^{2}(H e(t))^{\mathrm{T}} H e(t)}{\|H e(t)\|^{2}+\varepsilon \mathrm{e}^{-\sigma t}} .
\end{aligned}
$$

Here, we use the same method to deal with $\|e(t)\|^{2}$ as in the proof of Theorem 1. And if we design $k_{3}=0.5 l^{* 2}$, then $\dot{V}$ can be shown as

$$
\begin{aligned}
\dot{V} \leqslant-e(t)^{\mathrm{T}}\left(P \Lambda+\Lambda^{\mathrm{T}} P\right) e(t)+\frac{2 \beta(t)\|H e(t)\| \varepsilon \mathrm{e}^{-\sigma t}}{\beta(t)\|H e(t)\|+\varepsilon \mathrm{e}^{-\sigma t}}+ \\
\quad \frac{l^{*^{2}}\left\|e(t)^{\mathrm{T}} P B\right\|^{2} \varepsilon \mathrm{e}^{-\sigma t}}{\|H e(t)\|^{2}+\varepsilon \mathrm{e}^{-\sigma t}} .
\end{aligned}
$$

Using the similar approach as in the proof of Theorem 1, the second term of the right-hand side of (33) is equivalent to $c_{1} \mathrm{e}^{-\sigma t}$. Similarly, the third term of the right-hand side of (33) can be simplified as the form of

$$
\frac{l^{* 2}\left\|e(t)^{\mathrm{T}} P B\right\|^{2} \varepsilon \mathrm{e}^{-\sigma t}}{\|H e(t)\|^{2}+\varepsilon \mathrm{e}^{-\sigma t}}=\frac{l^{* 2}\left\|e(t)^{\mathrm{T}} P B\right\|^{2} \varepsilon \mathrm{e}^{-\sigma t}}{\|H e(t)\|^{2}} .
$$

Finally, (34) can be simplified as the form of

$$
\frac{l^{* 2} \varepsilon \mathrm{e}^{-\sigma t}}{\|W C\|^{2}}=c_{2} \mathrm{e}^{-\sigma t}
$$

where $\|W C\|^{2}$ and $l^{* 2}$ are positive constants and $\frac{\|W C\|^{2}}{l^{* 2}}$ is replaced by a real constant $c_{2}$. Therefore, $\dot{V}(t)$ satisfies the following inequality

$$
\dot{V} \leqslant-\lambda V+\left(c_{1}+c_{2}\right) \mathrm{e}^{-\sigma t} .
$$

And we define $P \Lambda+\Lambda^{\mathrm{T}} P=-Q, \lambda \triangleq\left\{\frac{\lambda_{\min }(Q)}{\lambda_{\max }(P)}\right\}>0$. On the basis of the comparison lemma, $V(t)$ satisfies that

$$
\begin{aligned}
& 0 \leqslant V(t) \leqslant \mathrm{e}^{-\lambda t} V(0)+ \\
& \quad \int_{0}^{t} \mathrm{e}^{-\lambda(t-\delta)}\left(c_{1}+c_{2}\right) \mathrm{e}^{-\sigma \delta} \mathrm{d} \delta, \forall t \geqslant 0 .
\end{aligned}
$$

Then, we denote $c_{1}+c_{2}=c$. The second term on the right-hand side of the above inequality can be

$$
\begin{aligned}
& \int_{0}^{t} \mathrm{e}^{-\lambda(t-\delta)} c \mathrm{e}^{-\sigma t} \mathrm{~d} \sigma= \\
& \begin{cases}c t \mathrm{e}^{-\lambda t}, & \text { if } \sigma \neq \lambda . \\
\frac{c}{\lambda-\sigma}\left(\mathrm{e}^{-\sigma t}-\mathrm{e}^{-\lambda t}\right), & \text { if } \sigma \neq \lambda .\end{cases}
\end{aligned}
$$


Obviously, we have $V(t) \rightarrow 0$ as $t \rightarrow \infty$, which indicates that $e(t) \rightarrow 0$ as $t \rightarrow \infty$.

Remark 6. $u_{\mathrm{des}}$ can be described as a function of $e(t)$ to make $\xi \rightarrow 0$ as $t \rightarrow \infty$, then we need to denote that $u_{\mathrm{des}}$ is also bounded by $\beta(t)$, i.e., $\left\|u_{\mathrm{des}}\right\|<\beta(t)$. From (36), increasing $\sigma$ or decreasing $c_{1}+c_{2}$ can accelerate the convergence of $V$. Furthermore, the selected parameter $\sigma$ should be larger than or equal to $\lambda$.

\section{$5 \quad$ Numerical example}

To illustrate the proposed controller design, a rocket fairing structural-acoustic model is considered by using the linear model with external disturbance input and parameter uncertainty. The details of state space matrix $A$, control input matrix $B$ and the known matrix $F_{d}$ associated with fault are shown as

$$
\begin{aligned}
A & =\left[\begin{array}{cccc}
0 & 1 & 0.0802 & 1.0415 \\
-0.1980 & -0.115 & -0.0318 & 0.3 \\
-3.0500 & 1.1880 & -0.4650 & 0.9 \\
0 & 0.0805 & 1 & 0
\end{array}\right] \\
B & =\left[\begin{array}{ccc}
1 & 1.55 & 0.75 \\
0.975 & 0.8 & 0.85 \\
0 & 0 & 0 \\
0 & 0 & 0
\end{array}\right] \\
F_{d} & =\left[\begin{array}{cc}
1.5 & 1 \\
-2 & -1 \\
-1 & 1
\end{array}\right] .
\end{aligned}
$$

In order to verify the effectiveness of the proposed adaptive method, the initial parameters are given as

$$
\begin{gathered}
x(0)=[0,1,0.5,-1]^{\mathrm{T}}, \hat{K}_{i}(0)=[0,0,0,0]^{\mathrm{T}}, \\
\hat{k}_{1}(0)=0, \quad \varepsilon=1, \quad \sigma=0.1, \quad \gamma=50, \Pi_{i}=10, \quad i=1,2,3, \\
S(t)=\left[\begin{array}{cccc}
0.5 \cos (t) & 0 & 0 & 0 \\
0 & 0 & 0 & 0 \\
0 & 0 & 0 & 0
\end{array}\right] .
\end{gathered}
$$

According to the proposed fault model, we fully consider the possible combinations of conditions to describe the fault model, the details of which are established in simulation as Table 1.

Table 1 Fault model

\begin{tabular}{cccc}
\hline Fault type & $\rho_{1}$ & $\rho_{2}$ & $\rho_{3}$ \\
\hline Normal & 1 & 1 & 1 \\
Fault mode 1 & 0 & $0.4-1$ & $0.6-1$ \\
Fault mode 2 & $0.5-1$ & 0 & $0.4-1$ \\
Fault mode 3 & $0.6-1$ & $0.5-1$ & 0 \\
\hline
\end{tabular}

The normal type implies that all the actuators are normally working, the fault modes from 1 to 3 describe that the first actuator, the second actuator and the third actuator are outage or suck, respectively. But the other actuators are normal or loss of effectiveness. Furthermore, $\Theta(t)$, which is supposed by the designed numerical function, is the indispensable part of fault model. The first fault is stuck at
$\Theta(t)=5+3 \sin (0.5 t)+2 \cos (0.5 t)$ and occurs at $8 \mathrm{~s}$, meanwhile we also consider that the second actuator has no fault during the whole operation process, and the third actuator faces loss of effectiveness by $1.5-0.03 \rho$. In other word, the system operates in a normal case before $8 \mathrm{~s}$. Moreover, the supposed external disturbance $d(t)=[1,0.5 \sin (4 t)]^{\mathrm{T}}$ enters into the system at the beginning $(t>0)$.

As illustrated in Fig. 1, the designed adaptive fault tolerant controller can guarantee that the state asymptotically converges to zero in the case that the actuator stuck fault is a time-varying signal under the unknown parameters. And then, the estimation parameters $\hat{K}$ and $\hat{k}_{1}$ for the controller are shown in Figs. $2-5$. It is easily seen that the estimations can converge and all the signals are uniformly bounded.

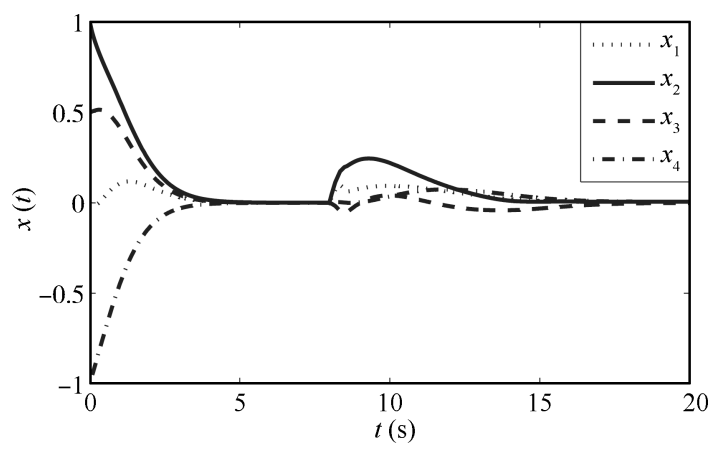

Fig. 1 Response curves of system state under the proposed control

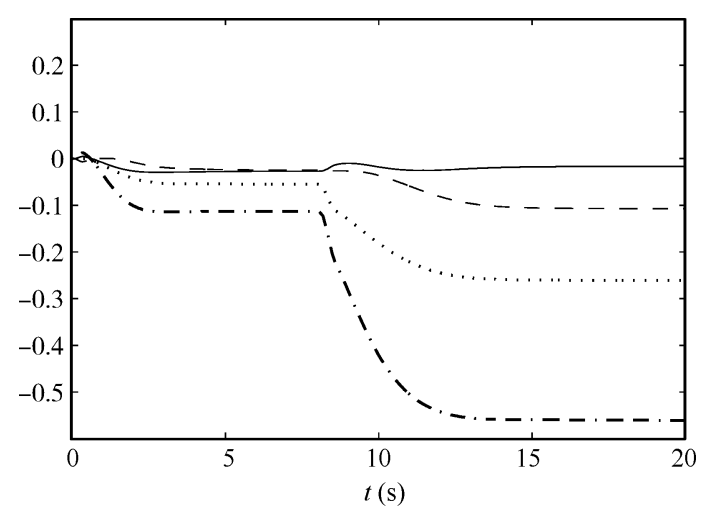

Fig. 2 Estimations of the first row of $\hat{K}$

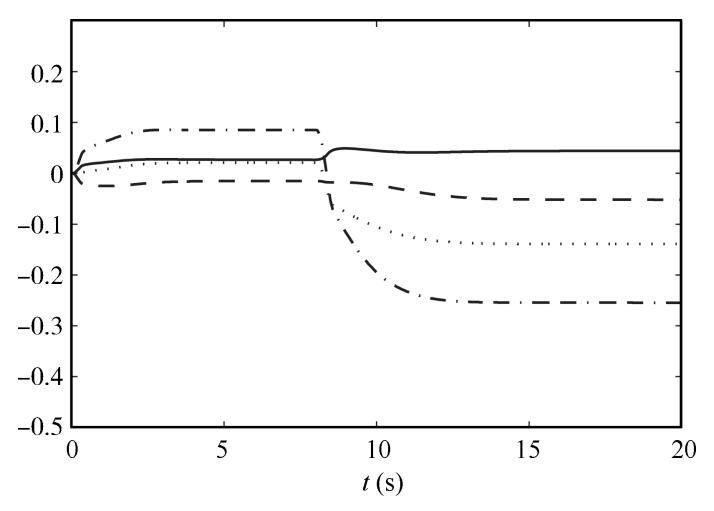

Fig. 3 Estimations of the second row of $\hat{K}$ 


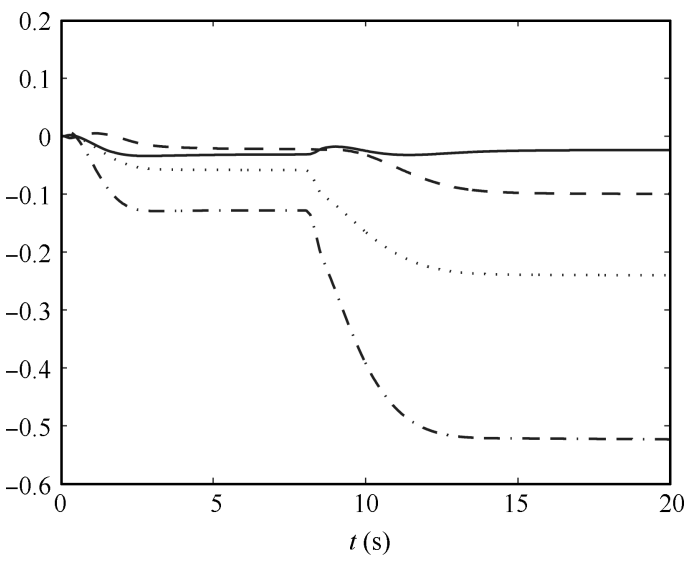

Fig. 4 Estimations of the third row of $\hat{K}$

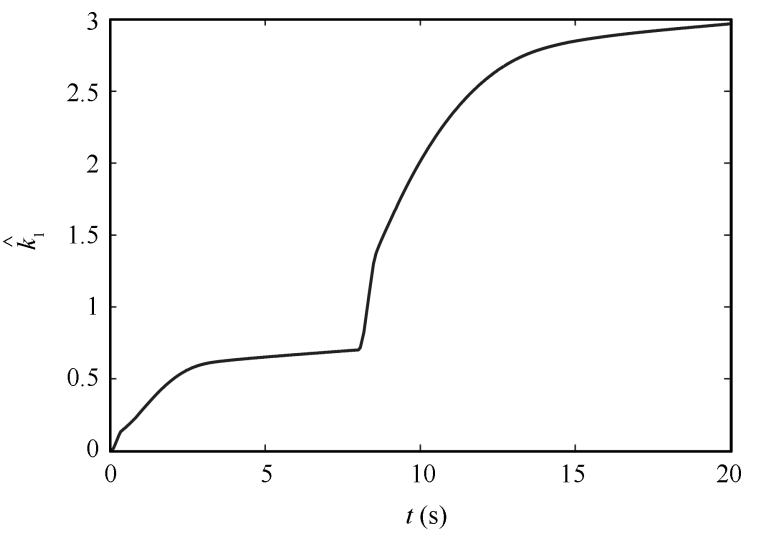

Fig. 5 The updating parameter $\hat{k}_{1}$

To decouple the disturbances and faults, we reconstruct the unknown external signals based on the observer method. The proposed matrix $L$, which is a feasible solution of (24), can be obtained by LMI. And we can simply choose an appropriate matrix $W=\left[I_{2}, 0_{2 \times 2} ; 0_{2 \times 2}, 0_{2 \times 2}\right]$ to ensure $W C \bar{B}>0$. Then $P$ can be obtained by the projection lemma in $[37,38]$ to satisfy SPR condition. Fig. 6 describes the actual fault input and the reconstruction external fault, the dummy fault signal $-u_{f}^{*}$ is designed to illustrate the efficient controller decoupling the faults and disturbances. Fig. 7 shows the waveforms of $d(t)$, the reconstruction of $d(t)$ and reconstruction error. The signal $e(t)$ is shown in Fig. 8.

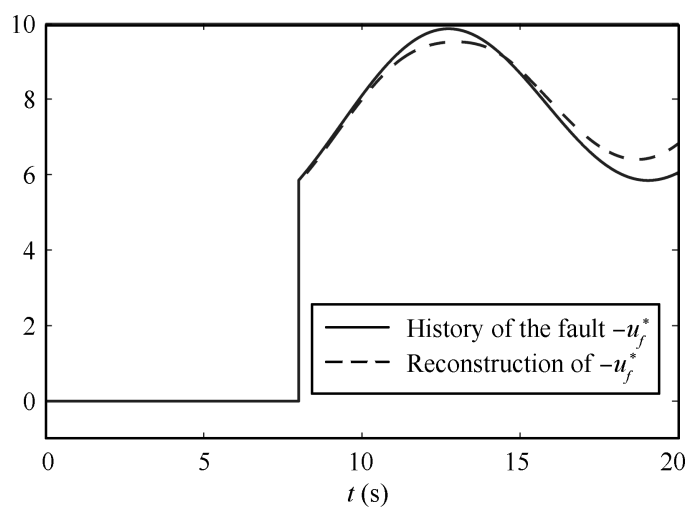

Fig. 6 History of the fault $-u_{f}^{*}$ and the reconstruction of $-u_{f}^{*}$
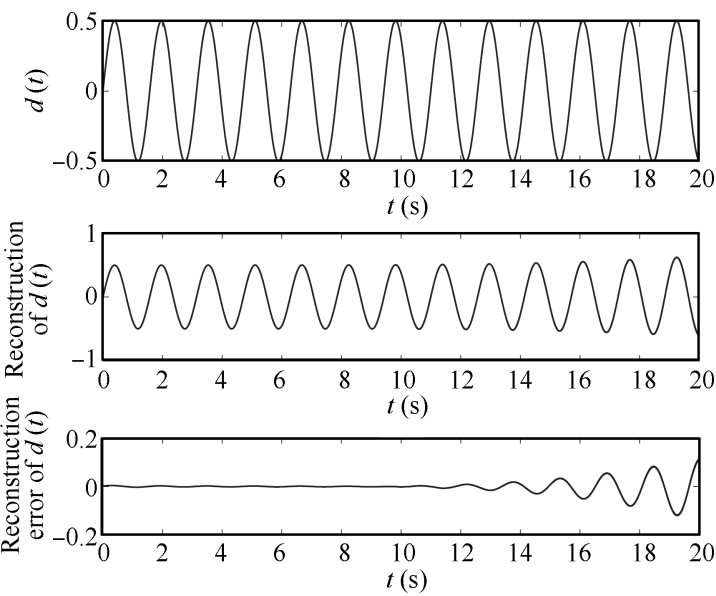

Fig. 7 Reconstruction of disturbance $d(t)$

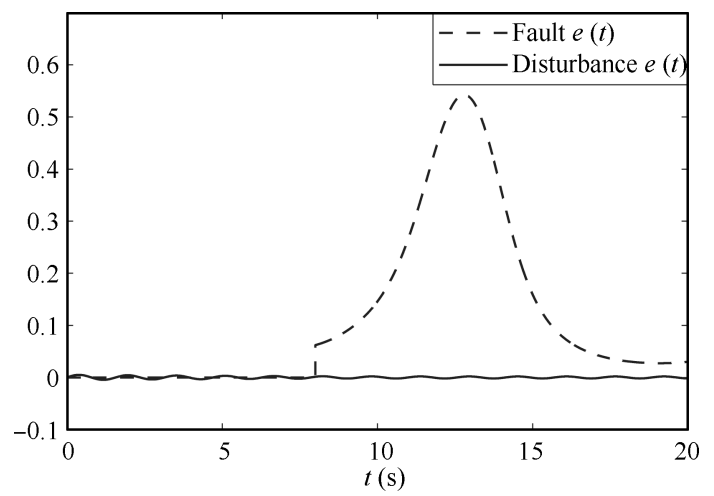

Fig. 8 The response curves of output error $e(t)$ with weight factor $W$

\section{Conclusions}

A robust adaptive output feedback with fault compensation control law was developed for linear systems subject to uncertainties, unknown disturbances and actuator failures. The presented active controller design scheme can deal with the development of the fault model and guarantee the states converge asymptotically to zero. And the Lyapunov redesign technique can be used to reconstruct the faults and disturbances. The advantage of this method can help to estimate the waveform of the fault and extend the design to fault accommodation.

\section{References}

[1] Y. M. Zhang, J. Jiang. Bibliographical review on reconfigurable fault-tolerant control systems. Annual Reviews in Control, vol. 32, no. 2, pp. 229-252, 2008.

[2] Q. Zhao, J. Jiang. Reliable state feedback control system design against actuator failures. Automatica, vol. 34, no. 10, pp. 1267-1272, 2006.

[3] R. J. Veillette. Reliable linear-quadratic state-feedback control. Automatica, vol. 31, no. 1, pp. 137-143, 1995. 
[4] M. J. Khosrowjerdi, R. Nikoukhah, N. Safari-Shad. A mixed $H_{2} / H_{\infty}$ approach to simultaneous fault detection and control. Automatica, vol. 40, no. 2, pp. 261-267, 2004.

[5] Z. C. Wang, Y. T. Wen, X. Y. Luo. Quantized $H_{\infty}$ faulttolerant control for networked control systems. International Journal of Automation and Computing, vol. 9, no. 4, pp. 352-357, 2012.

[6] Y. M. Zhang, J. Jiang. Active fault tolerant control system against partial actuator failures. IEEE Proceedings Control Theory and Applications, vol.149, no. 1, pp.95104, 2002.

[7] M. Wang, D. H. Zhou. Fault tolerant control of feedback linearizable systems with stuck actuators. Asian Journal of Control, vol. 10, no. 1, pp. 74-87, 2008.

[8] G. J. Liu, Y. C. Li, D. J. Wang. Active fault tolerant control with actuation reconfiguration. IEEE Transactions on Aerospace and Electronic Systems, vol. 40, no. 3, pp. 11101117,2004

[9] S. Y. Cao, L. Guo, X. Y. Wen. Fault tolerant control with disturbance rejection and attenuation performance for systems with multiple disturbances. Asian Journal of Control, vol. 13, no. 6, pp. 1056-1064, 2011.

[10] O. Härkegard, S. T. Glad. Resolving actuator redundancyoptimal control vs. control allocation. Automatica, vol. 41, no. 1, pp. 137-144, 2005.

[11] H. Alwi, C. Edwards. Fault tolerant control using sliding modes with on-line control allocation. Automatica, vol. 44, no. 7 , pp. 1859-1866, 2008.

[12] J. D. Boskovic, R. K. Mehra. Control allocation in overactuated aircraft under position and rate limiting. In Proceedings of the American Control Conference, IEEE, Anchorage, AK, USA, pp. 791-796, 2002.

[13] C. Edwards, S. K. Spurgeon, R. J. Patton. Sliding mode observers for fault detection and isolation. Automatica, vol. 36, no. 4, pp. 541-553, 2000.

[14] C. P. Tan, F. Crusca, M. Aldeen. Extended results on robust state estimation and fault detection. Automatica, vol.44, no. 8, pp. 2027-2033, 2008.

[15] X. L. Wu, X. J. Wu, X. Y. Luo, Q. M. Zhu. Design of observer-based adaptive controller for nonlinear systems with unmodeled dynamics and actuator dead-zone. International Journal of Automation and Computing, vol. 8, no. 2, pp. 201-208, 2011.

[16] D. Ye, G. H. Yang. Adaptive fault-tolerant tracking control against actuator faults with application to flight control. IEEE Transactions on Control Systems Technology, vol. 14, no. 6, pp. 1088-1096, 2006.
[17] G. H. Yang, D. Ye. Reliable $H_{\infty}$ control of linear systems with adaptive mechanism. IEEE Transactions on $\mathrm{Au}$ tomatic Control, vol. 55, no. 1, pp. 242-247, 2010.

[18] Z. Q. Zuo, D. W. C. Ho, Y. J. Wang. Fault tolerant control for singular systems with actuator saturation and nonlinear perturbation. Automatica, vol. 46, no. 3, pp. 569-576, 2010.

[19] X. D. Tang, G. Tao, L. F. Wang, J. A. Stankovic. Robust and adaptive actuator failure compensation designs for a rocket fairing structural-acoustic model. IEEE Transactions on Aerospace and Electronic Systems, vol. 40, no. 4, pp. 1359-1366, 2004.

[20] G. Tao, S. M. Joshi, X. L. Ma. Adaptive state feedback and tracking control of systems with actuator failures. IEEE Transactions on Automatic Control, vol.46, no. 1, pp. 7895, 2001.

[21] W. Wang, C. Y. Wen. Adaptive actuator failure compensation control of uncertain nonlinear systems with guaranteed transient performance. Automatica, vol. 46, no. 12, pp. 2082-2091, 2010.

[22] X. Z. Jin, G. H. Yang. Robust adaptive fault-tolerant compensation control with actuator failures and bounded disturbances. Acta Automatica Sinica, vol. 35, no. 3, pp. 305$309,2009$.

[23] J. D. Boskovic, R. K. Mehra. A decentralized fault-tolerant control system for accommodation of failures in higherorder flight control actuators. IEEE Transactions on Control Systems Technology, vol. 18, no. 5, pp. 1103-1115, 2010.

[24] J. Li, G. Y. Tang, P. Zhang, J. Zou. Observer-based fault diagnosis and self-restore control for systems with measurement delays. Asian Journal of Control, vol. 14, no. 6, pp. 1717-1723, 2012.

[25] J. Li, G. Y. Tang, P. Zhang, D. R. Huang. Fault diagnosis and optimal fault-tolerant control for systems with delayed measurements and states. International Journal of Control, Automation, and Systems, vol. 10, no. 1, pp. 150-157, 2012.

[26] K. Zhang, B. Jiang, P. Shi. Fast fault estimation and accommodation for dynamical systems. IET Control Theory and Application, vol. 3, no. 2, pp. 189-199, 2009.

[27] W. Chen, M. Saif. Adaptive actuator fault detection, isolation and accommodation in uncertain systems. International Journal of Control, vol. 80, no. 1, pp. 45-63, 2007.

[28] H. Wang, S. Daley. Actuator fault diagnosis: An adaptive observer-based technique. IEEE Transactions on Automatic Control, vol. 41, no. 7, pp. 1073-1078, 1996.

[29] R. J. Patton, S. Klinkhieo. Actuator fault estimation and compensation based on an augmented state observer approach. In Proceedings of the 48th IEEE Conference on Decision and Control and 28th Chinese Control Conference, IEEE, Shanghai, China, pp. 8482-8487, 2009. 
[30] Z. Gao, S. X. Ding. Fault estimation and fault-tolerant control for descriptor systems via proportional, multipleintegral and derivative observer design. IET Control Theory and Applicationa, vol. 1, no. 5, pp. 1208-1218, 2007.

[31] M. Corless, J. Tu. State and input estimation for a class of uncertain systems. Automatica, vol. 34, no. 6, pp. 757-764, 1998.

[32] J. D. Boskovic, R. K. Mehra. Stable multiple model adaptive flight control for accommodation of a large class of control effector failures. In Proceedings of the American Control Conference, IEEE, San Diego, CA, USA, pp. 19201924, 1999

[33] J. J. Slotine, W. P. Li. Applied Nonlinear Control, New Jersey, USA: Prentice Hall, pp. 122-123, 1991.

[34] H. S. Wu. Adaptive robust tracking and model following of uncertain dynamical systems with multiple time delays. IEEE Transactions on Automatic Control, vol.49, no. 4, pp. 611-616, 2004.

[35] U. Maeder, F. Borrelli, M. Morari. Linear offset-free model predictive control. Automatica, vol.45, no. 10, pp. 2214$2222,2009$.

[36] H. K. Khalil. Nonlinear System, New Jersey, USA: Prentice Hall, pp. 156-174, 2002.

[37] P. Gahinet, P. Apkarian. A linear matrix inequality approach to $H_{\infty}$ control. International Journal of Robust and Nonlinear Control, vol. 4, no. 4, pp.421-448, 1994.

[38] T. Iwasaki, R. E. Skelton. All controllers for the general $H_{\infty}$ control problem: LMI existence conditions and state space formulas. Automatica, vol. 30, no. 8, pp. 1307-1317, 1994.

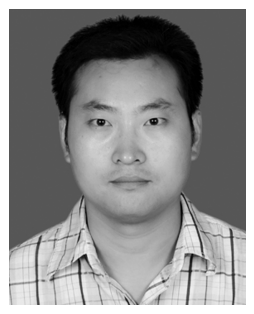

Jun Wang received his B.Sc. and M. Sc. degrees form Zhengzhou University of Light Industry, China in 2007 and 2010, respectively. He is currently a $\mathrm{Ph}$. D. candidate at College of Automation Science and Engineering, South China University of Technology, China.

His research interests include robust fault-tolerant control and optimal control algorithm.

E-mail: jwangsunny@gmail.com

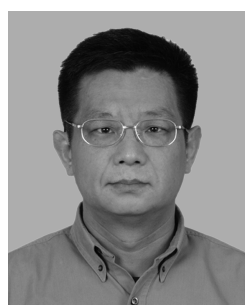

Hai-Long Pei received his B.Sc. and M. Sc. degrees from Northwestern Polytechnical University, China in 1986 and 1989 and his $\mathrm{Ph}$. D. degree from College of $\mathrm{Au}-$ tomation Science and Engineering, South China University of Technology, China in 1992. From 1997 to 1998, he was a postdoctoral fellow at the Chinese University of Hong Kong. From 1999 to 2001, he worked as a senior visiting scholar at Carnegie Mellon University, USA. Currently, he is a professor of South China University of Technology, China.

His research interests include robust control, nonlinear control, embedded system, computer numerical control system, and artificial neural network.

E-mail: auhlpei@scut.edu.cn (Corresponding author)

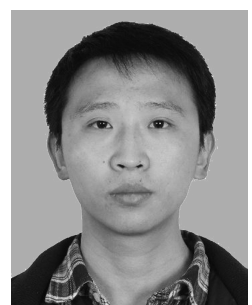

Nai-Zhou Wang received his B.Sc. degree from Jiangnan University, China in 2007 and his M.Sc. degree from Nanjing Forestry University, China in 2010. He is currently a Ph. D. candidate at College of Automation Science and Engineering, South China University of Technology, China.

His research interests include robust and optimal control, especially the anti-windup control methods.

E-mail: wangnzhou@sina.com 